\title{
Epidemiology, clinical differences and outcomes of tracheobronchitis and pneumonia associated to mechanical ventilation in intensive care units of latin america (LATINNAVE)
}

\author{
A Ali Munive ${ }^{1}$, FA Varon Vega ${ }^{2 *}$, A Hernandez Parra ${ }^{1}$, F Molina ${ }^{1}$, M Poveda ${ }^{1}$, RA Meza ${ }^{1}$, H Castro ${ }^{1}$, Z Urbina ${ }^{1}$, \\ J Mercado ${ }^{1}$, J Martinez ${ }^{1}$, M Mayorga ${ }^{1}$, M Pareja ${ }^{1}$, E Cepeda ${ }^{1}$, M Sanchez ${ }^{1}$, R Vega', F Camargo ${ }^{1}$, J Vergara ${ }^{1}$
}

From ESICM LIVES 2015

Berlin, Germany. 3-7 October 2015

\section{Introduction}

The infections associated with mechanical ventilation are a major cause of morbidity and mortality in critically ill patients. Limited studies report increased mortality and ICU stay, requirement for mechanical ventilation and higher costs in ventilator-associated Tracheobronchitis (TAV) in comparison to patients with ventilatorassociated pneumonia (NAV). These studies do not describe the clinical and epidemiological behavior in the same population as independent entities, so it is necessary to describe the epidemiology of patients with TAV and NAV.

\section{Methodology}

Multicenter cross-sectional study of adult patients who developed TAV and / or NAV during their stay in the ICU in 2013 to 2014. Each of the variables was performed descriptive analysis; to assess differences between the groups using Chi-square qualitative variables; in continuous variables, $\mathrm{t}$ of Student's have normal distribution; otherwise the Mann Whitney U test.

\section{Results}

Latin Nave collected 147 patients from 6 countries in Latin America; 63\% with NAV and 37\% with TAV. The average age was 51 years; $57 \%$ male. The most frequent comorbidity was cardiovascular (44\%) and neurological (30\%), the later higher in TAV (41.5 Vs $23 \%$, p 0.02$)$. No difference was found in APACHE II at admission,

${ }^{2}$ Fundación Neumológica Colombiana, Critical Care, Bogotá, Colombia Full list of author information is available at the end of the article but the difference appears in SOFA ( 8 vs. $5, \mathrm{p} 0.02$ ). There were no differences in microbiological isolation, or bacterial resistance patterns between the two entities. Greater number of cardiovascular complications and ARDS were observed in patients with NAV. The ICU stay, days of mechanical ventilation and mortality were not different between the two groups.

\section{Conclusions}

TAV prevalence was higher than heretofore described in the literature, indicating that it is considered an independent clinical entity. No significant differences were found in the microbiological isolation, bacterial resistance and antibiotic therapy used in the two groups, which may suggest that therapeutic approach may be similar to that recommended for NAV. No differences were observed in clinical outcomes such as hospital stay, duration of mechanical ventilation and mortality, although the NAV was associated with greater proportion of medical complications.

\section{Authors' details}

${ }^{1}$ Fundación Neumológica Colombiana, Bogotá, Colombia. ${ }^{2}$ Fundación Neumológica Colombiana, Critical Care, Bogotá, Colombia.

Published: 1 October 2015

doi:10.1186/2197-425X-3-S1-A703

Cite this article as: Ali Munive et al.: Epidemiology, clinical differences and outcomes of tracheobronchitis and pneumonia associated to mechanical ventilation in intensive care units of latin america (LATINNAVE). Intensive Care Medicine Experimental 2015 3(Suppl 1):A703.

\section{SpringerOpen ${ }^{\odot}$}

(c) 2015 Varon Vega et al.; This is an Open Access article distributed under the terms of the Creative Commons Attribution License (http://creativecommons.org/licenses/by/4.0), which permits unrestricted use, distribution, and reproduction in any medium, provided the original work is properly cited. 